\title{
BMJ Open Symptom heterogeneity and patient subgroup classification among US patients with post-treatment Lyme disease: an observational study
}

\author{
Alison W Rebman (D), Ting Yang, John N Aucott
}

To cite: Rebman AW, Yang T, Aucott JN. Symptom heterogeneity and patient subgroup classification among US patients with posttreatment Lyme disease: an observational study. BMJ Open 2021;11:e040399. doi:10.1136/ bmjopen-2020-040399

- Prepublication history and additional material for this paper is available online. To view these files, please visit the journal online (http://dx.doi.org/10. 1136/bmjopen-2020-040399).

AWR and TY contributed equally.

Received 12 May 2020

Revised 25 November 2020

Accepted 07 December 2020

Check for updates

(c) Author(s) (or their employer(s)) 2021. Re-use permitted under CC BY-NC. No commercial re-use. See rights and permissions. Published by BMJ.

Lyme Disease Research Center, Division of Rheumatology, Department of Medicine, Johns Hopkins University School of Medicine, Baltimore, Maryland, USA

Correspondence to

Dr John N Aucott;

jaucott2@jhmi.edu

\section{ABSTRACT}

Objectives To identify underlying subgroups with distinct symptom profiles, and to characterise and compare these subgroups across a range of demographic, clinical and psychosocial factors, within a heterogeneous group of patients with well-defined post-treatment Lyme disease (PTLD).

Design A clinical case series of patents.

Setting Participants were recruited from a single-site, Lyme disease referral clinic patient population and were evaluated by physical exam, clinical laboratory testing and standardised questionnaires.

Participants Two hundred and twelve participants met study criteria for PTLD, with medical record-confirmed prior Lyme disease as well as current symptoms and functional impact.

Results Exploratory factor analysis classified 30 selfreported symptoms into 6 factors: 'Fatigue Cognitive', 'Ocular Disequilibrium', 'Infection-Type', 'Mood-Related', 'Musculoskeletal Pain' and 'Neurologic'. A final latent profile analysis was conducted using 'Fatigue Cognitive', 'Musculoskeletal Pain' and 'Mood-Related' factor-based scores, which produced three emergent symptom profiles, and participants were classified into corresponding subgroups with $59.0 \%, 18.9 \%$ and $22.2 \%$ of the sample, respectively. Compared with the other two groups, subgroup 1 had similarly low levels across all factors relative to the sample as a whole, and reported lower rates of disability $(1.6 \%$ vs $10.0 \%, 12.8 \% ; q=0.126,0.035)$ and higher self-efficacy (median: 7.5 vs $6.0,5.3 ; q=0.068,<0.001$ ). Subgroup 2 had the highest 'Musculoskeletal Pain' factor-based scores $(q \leq 0.001)$. Subgroup 3 was characterised overall by higher symptom factor-based scores, and reported higher depression $(\mathrm{q} \leq 0.001)$.

Conclusions This analysis identified six symptom factors and three potentially clinically relevant subgroups among patients with well-characterised PTLD. We found that these subgroups were differentiated not only by symptom phenotype, but also by a range of other factors. This may serve as an initial step towards engaging with the symptom heterogeneity that has long been observed among patients with this condition.

\section{INTRODUCTION}

Lyme disease is a tick-borne disease of increasing public health importance found primarily across temperate regions of the
Strengths and limitations of this study

- We operationalised a rigorous definition of posttreatment Lyme disease (PTLD) in our sample population, which ensured greater specificity of our findings to patients whose current illness is more evidently linked to prior Lyme disease.

- This specificity, and the regional focus of our sample population, may limit generalisability to the larger population of patients with persistent symptoms following treatment for Lyme disease, or those from other regions of the USA.

- Reproducibility of the subgroup analysis may be affected by necessary methodological decisions incorporating statistical and clinical criteria which were made during the analytic process.

- We were able to draw on a relatively large sample size of participants with well-characterised PTLD, which allowed for clear and concise interpretability of data.

Northern Hemisphere. ${ }^{12}$ Clinical signs of early infection may include a round, red, skin lesion occurring at the site of the bite of infected Ixodes ticks, and/or a transient, nonspecific illness consisting of fever, fatigue, myalgia or arthralgia. ${ }^{3}$ If not promptly identified or otherwise left untreated, the bacteria (Borrelia burgdorferi in the USA) can disseminate to other areas of the skin, and via the blood stream to other organs such as the nervous system, heart and joints. ${ }^{4}$ Consequently, although less commonly observed, patients with untreated infection can present with objective, later manifestations of neurologic disease, carditis or arthritis. ${ }^{3}$

While the majority of patients treated appropriately for Lyme disease recover, a subset develop a poorly understood, chronic illness of persistent or recurrent symptoms following treatment. ${ }^{5}$ The presence of chronic or persistent symptoms following acute infection has been documented in a subset of patients for a number of viral and 
bacterial pathogens. ${ }^{6}$ Although more research is needed, the symptom phenotype of these illnesses, including that of the newly described 'long COVID-19' shares many overlapping characteristics. ${ }^{67}$ In order to methodically advance scientific understanding, a standardised, highly specific, research definition for post-treatment Lyme disease (PTLD, alternatively previously called posttreatment Lyme disease syndrome or PTLDS) has been used and operationalised to identify a subset of these patients with on-going symptoms linked temporally to strong evidence of prior exposure to B. burgdorferi. ${ }^{389}$ The most prominent symptoms, and those included in the Infectious Diseases Society of America's (IDSA) proposed case definition of PTLD, ${ }^{3}$ include fatigue, musculoskeletal pain and cognitive dysfunction. However, patients with PTLD often also report a broad range of other neurologic, sleep, mood, ocular and other symptoms. ${ }^{81011}$ This heterogeneity is often compounded by the significant impact of these symptoms on patient quality of life and functioning. ${ }^{8}{ }^{12}$ Additionally, given the lack of: (a) a sensitive and specific test to aid diagnosis, (b) The United States Food and Drug Administration-approved treatment options for patients and (c) a known aetiology, PTLD presents a complex challenge to physicians.

As large studies among patients with well-characterised PTLD have not been conducted, this diversity in PTLD symptom reporting has not been comprehensively examined and it is unknown whether it may obscure the presence of distinct clinical patient subgroups. However, it is increasingly common that through advances in personalised medicine, diseases previously considered a single entity have been found instead to be comprised of clinically and/or biologically coherent subgroups. ${ }^{13} 14$ Furthermore, similar to fibromyalgia, PTLD is likely a complex, multifactorial illness with immunologic, microbiologic, genetic and/or psychosocial factors contributing to disease development, severity and persistence. ${ }^{5}{ }^{15}$ Consequently, examining the heterogeneity of clinical presentations and symptom reporting that exists among patients with PTLD is important because it may inform a deeper understanding of aetiology and effective treatment approaches. Therefore, the aims of this observational study were (a) to identify underlying patient subgroups with distinct symptom profiles within a heterogeneous group of patients with well-defined PTLD and (b) to characterise and compare these subgroups across a range of demographic, clinical, laboratory and psychosocial factors.

\section{METHODS}

\section{Study participants}

Participants were recruited from a referral-based clinic population. Detailed recruitment information and enrolment criteria for this study were included in an initial publication describing a subset of the larger sample of participants included in the current analysis. ${ }^{8}$ In brief, we replicated much of the criteria set forth in the IDSA's proposed case definition for PTLD through our enrollment criteria. ${ }^{38}$ Participants were required to have prior evidence in their medical record of appropriately treated, Centers for Disease Control and Prevention (CDC)definite or probable Lyme disease. ${ }^{16}$ They were also required to have current, functionally impairing fatigue, pain and/or cognitive dysfunction, and were excluded for a range of specific comorbid medical conditions, as previously described. ${ }^{8}$ For the current analysis, we did not limit the sample to those with greater than 6 month's illness duration, and thus, we refer to our sample as meeting criteria for PTLD. The Institutional Review Board of the Johns Hopkins University School of Medicine approved this study, and written informed consent was obtained from all study participants.

\section{Patient and public involvement}

Patients and the public were not directly involved in the design, recruitment or assessment of this study.

\section{Data collection instruments}

Participants were asked to self-administer a 36-item post-Lyme questionnaire of symptoms (PLQS) developed based on prior clinical and research experience among patients with PTLD. ${ }^{8}$ Participants indicated both presence and severity over the past 2 weeks for each symptom ( $0=$ absent, $1=$ mild, $2=$ moderate or $3=$ severe $)$. Of the original 36 symptoms, we excluded the following, which occurred with low frequency in our sample and were not considered to be core symptoms of PTLD (the per cent endorsed at a moderate or severe level): urination pattern change $(9 \%)$, diarrhoea $(8 \%)$, sore throat $(5 \%)$, drooping eyelid(s) (2\%), Bell's palsy $(1 \%)$ and tender lymph nodes (2\%). Data from the remaining 30 symptoms provided the basis for the subgroup analyses described below (see online supplemental table 1 for the complete list of symptoms).

Participants were also asked to self-administer a battery of additional questionnaires included in the current analyses. The Beck Depression Inventory-II is a 21-item depression metric which can be divided into 'Somatic' and 'Cognitive-Affective' subscales. ${ }^{17}{ }^{18}$ In order to avoid duplication with other variables in this analysis, only the 'Cognitive-Affective' subscale of the Beck Depression Inventory-II (BDI-C/A) was included, which has a total score of 0-48. Quality of life was measured by the ShortForm Health Survey, Version 2 (SF-36). ${ }^{19}$ This 36-item metric can be summarised into Physical and Mental Component Scores (PCS and MCS, respectively), with a higher score indicating higher quality of life. These scores can also be compared with the US population mean $(50.0 \pm 10.0)$. The Life Events Checklist (LEC) is a 17 -item measure with total scores of $0-17$ of prior potentially traumatic events originally developed to aid in the diagnosis of post-traumatic stress disorder. ${ }^{20}$ The Stanford Chronic Disease Self-Efficacy Scale (CDSE) is a 6-item measure of perceived self-efficacy for chronic disease self-management. ${ }^{21}{ }^{22}$ The Big Five Inventory (BFI) is a 
44-item measure of five personality dimensions; extraversion, agreeableness, conscientiousness, emotional stability and openness. ${ }^{23-25}$ Variables related to prior, initial Lyme disease clinical presentation, treatment(s) and duration of illness were abstracted from participants' medical records from the time of Lyme disease onset. Participants self-reported other prior medical diagnoses as part of a structured clinical interview.

During the study visit, a physical exam was performed which included routine measures of height, weight, pulse and blood pressure. Body mass index was calculated using the standard formula (weight $(\mathrm{kg}) /$ height $\left(\mathrm{m}^{2}\right)$ ). Vibratory index was measured on the distal interphalangeal joint of the index finger and on the interphalangeal joint of the hallux using a Rydel-Seiffer $64 \mathrm{~Hz}$ tuning fork. ${ }^{26}$ Lastly, participants underwent a blood draw, and standard clinical tests (complete blood count, comprehensive metabolic panel, $\mathrm{C}$ reactive protein and two-tier serology for antibodies to B. burgdorferi) were performed by a large, commercial laboratory.

\section{Statistical analysis}

We hypothesised that subcollections of symptoms are caused by different but interrelated underlying biological mechanisms, which are not directly observable in our study.

Therefore, we first performed exploratory factor analysis (EFA) to identify the latent relational structure of the symptoms included in the PLQS, which subsequently also reduced the dimensionality of the data. The KaiserMeyer-Olkin measure of sampling adequacy and Bartlett's test of sphericity were used to check whether the data were suitable for factor analysis. Considering the ordinal nature of the variables, both polychoric and Pearson's correlation coefficients were used. We chose the minimal residual estimation method because it can be used when the sample size is relatively small and when the correlation matrix is non-positive definite. ${ }^{27}$ Oblique rotation was used to allow for correlations between extracted factors. The number of retained factors was informed by the visual scree test and parallel analysis, while taking into consideration clinical meaningfulness and the balance between parsimony and comprehensiveness. We used a factor loading cut-off value of 0.3 .

Next, to uncover subgroups of participants, we performed latent profile analysis (LPA) on the standardised symptom factor-based scores generated by the EFA. The number of identified clusters was determined based on minimisation of the Bayesian information criteria and the correlational structure of the data. Lastly, pairwise subgroup differences were examined and summarised using two-sample $\mathrm{t}$ test or Wilcoxon rank sum test for continuous variables and $\chi^{2}$ or Fisher's exact test for categorical variables. Considering the accumulation of type 1 error across multiple hypothesis tests, we calculated $q$ values to control false discovery rate at $5 \%{ }^{28}$ All statistical analyses were performed using R (V.3.6.1).
Table 1 Characteristics of 212 participants with welldefined post-treatment Lyme disease*

All participants $\mathrm{n}=\mathbf{2 1 2}$

\begin{tabular}{ll}
\hline Age at study visit & $\begin{array}{l}48.00(37.00,58.00) \\
(18.00,82.00)\end{array}$ \\
\hline Male gender & $124(58.5 \%)$ \\
\hline $\begin{array}{l}\text { White, non-Hispanic } \\
\text { Years of education }\end{array}$ & $190(89.6 \%)$ \\
& $(10.00(14.00,18.00)$ \\
\hline Annual household income >US\$100K & $119 / 203(58.6 \%)$ \\
\hline Currently out of work on disability & $12(5.7 \%)$ \\
\hline $\begin{array}{l}\text { Lyme disease onset while resident of } \\
\text { CDC Lyme disease 'high-incidence' }\end{array}$ & $205(96.7 \%)$ \\
state & \\
\hline $\begin{array}{l}\text { CDC 'confirmed' initial Lyme disease } \\
\text { presentation }\end{array}$ (6) & $124(58.5 \%)$ \\
\hline $\begin{array}{l}\text { Duration from Lyme disease onset to } \\
\text { study visit (years) }\end{array}$ & $1.67(0.68,3.81)$ \\
\hline $\begin{array}{l}\text { Total antibiotic exposure from Lyme } \\
\text { disease onset to study visit (weeks) }\end{array}$ & $\begin{array}{l}8.57(4.43,14.29) \\
(2.00,168.57)\end{array}$ \\
\hline
\end{tabular}

*Data from categorical variables are presented as count (\%). Data from normally distributed continuous variables are presented as mean $\pm S D$ (range) and from continuous variables not normally distributed as median (25th percentile, 75th percentile) (range). Proportions were calculated based on non-missing data and may not add to $100 \%$ because of rounding. Missing data are as follows: Years of education, 1 (0.5\%); Annual household income, 9 (4.2\%). CDC, Centers for Disease Control and Prevention; PTLD, posttreatment Lyme disease.

\section{RESULTS}

\section{Participant characteristics}

A total of 225 participants with PTLD were enrolled in the study. We excluded six participants whose PTLD symptoms began more than 6 months after their initial Lyme disease episode, and seven participants who missed all symptom variables on the PLQS, for a total of 212 in the final sample. We employed mean imputation for three participants who each missed 1 of the 30 PLQS variables included in the analysis. Table 1 shows a description of the final participant sample. The average age was 48 years and there was a slight $(58.5 \%)$ majority male in the sample. A large majority were residents of Mid-Atlantic states at the time of their disease onset $(93.4 \%)$ and/or residents of states considered 'high-incidence' for Lyme disease $(96.7 \%){ }^{29}$

\section{Latent relational structure among symptoms}

The total symptom score among patients with PTLD ranged from 2 to 70 , with a median and first and third quartile interval of $22(14,33)$. Histograms of individual symptom scores are presented in online supplemental figure 1. In the EFA analysis, the original polychoric correlation matrix was non-positive definite. After smoothing was performed to arrive at a positive definite 


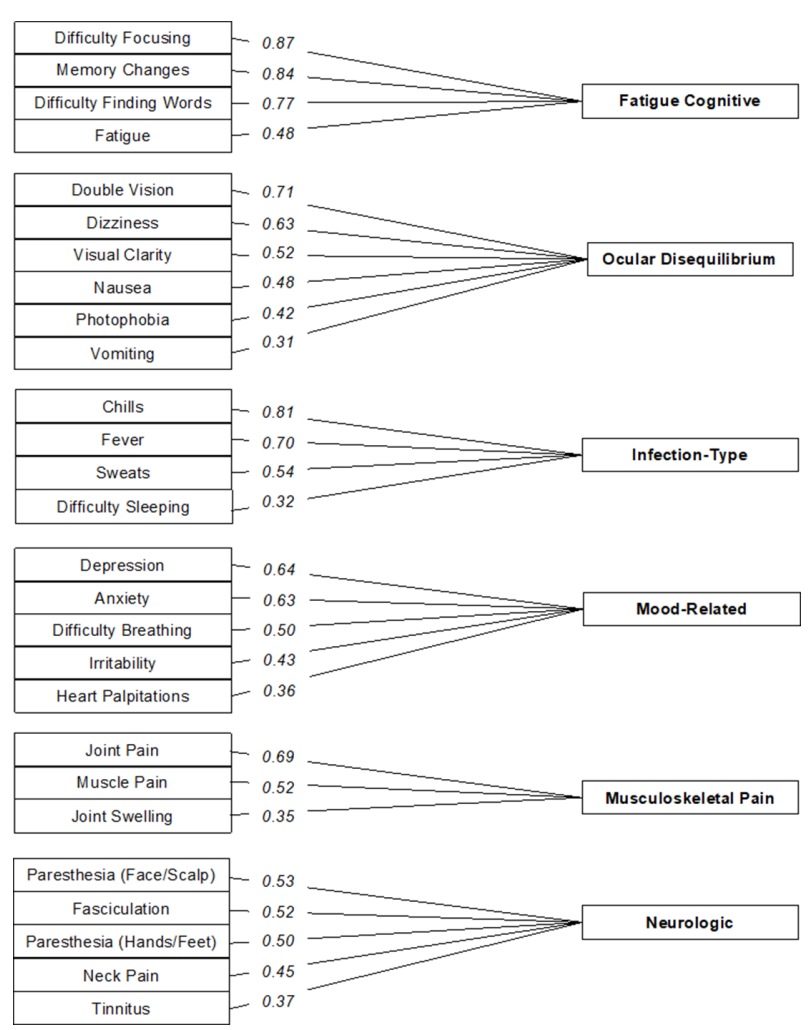

Figure 1 Exploratory factor analysis of 30 common posttreatment Lyme disease syndrome symptoms suggests a six-factor model. Three of the symptoms either did not load or loaded weakly and had close cross-loading, and they were not included in the final model.

matrix, it resulted in a poor overall sampling adequacy index $(0.10)$ and an ultra-Heywood case was detected. However, the overall measure of sampling adequacy based on the Pearson's correlation coefficient was 0.86 (meritorious), and Bartlett's test of sphericity was significant $(\mathrm{p}<0.001)$. A six-factor model was suggested by both statistical criteria and clinical meaningfulness (figure 1, see online supplemental table 1 for the complete factor pattern matrix). The root mean square of the residuals was 0.04 , the root mean square error of approximation index was 0.06 and the Tucker Lewis Index of factoring reliability was 0.85 . The symptom headache did not significantly load to any factor (maximum loading: 0.22, online supplemental table 1). Poor coordination and lower back pain loaded weakly to multiple factors (maximum loading $\leq 0.33$ ), and had close cross loading (difference less than $0.10)$ across two or more factors, and were therefore removed. The percent endorsed at a moderate or severe level for these symptoms was $34.9 \%, 15.6 \%$ and $35.8 \%$, respectively. An expert physician on the study team (JNA) named the factors as 'Fatigue Cognitive', 'Ocular Disequilibrium', 'Infection-Type', 'Mood-Related', 'Musculoskeletal Pain' and 'Neurologic'. All six factors were weakly or moderately correlated with each other $(0.21-0.41)$, with the strongest correlation between the 'Fatigue Cognitive' and 'Mood-Related' factors. For a more straightforward interpretation, six factor-based scores were calculated for each participant by adding up the scores of the symptoms within each factor, and then these factor-based scores were standardised to have a mean of 0 and an SD of 1.

\section{Participant subgroup analysis}

For the LPA analysis, we did not include the 'Ocular Disequilibrium' factor as it prevented the LPA from converging for most of the specified models in model selection, possibly due to its low endorsement rate (the percentage endorsing symptoms included in this factor at a moderate or severe level ranged from $0.9 \%$ to $24.1 \%$ ). When conducted on the remaining five factors, LPA classified participants into two groups based on their overall level of symptom reporting (high vs low) relative to the sample as a whole.

We then conducted a secondary LPA incorporating those factors which contained only the most common PTLD-defining symptoms as well as mood (ie, 'Fatigue Cognitive', 'Musculoskeletal Pain' and 'Mood-Related'). Three symptom profiles emerged (figure 2) and participants were classified into subgroups corresponding to these symptom profiles. Subgroup 1 contained $59.0 \%$ of the participants and was characterised by similarly low levels across all three factors relative to the sample as a whole. Subgroups 2 and 3 contained $18.9 \%$ and 22.2\% of the participants, respectively, and were characterised by overall higher levels of the three factors relative to the entire sample. These results remained stable when the 'Neurologic' factor was reintroduced in the LPA.

\section{Participant subgroup comparisons}

We first compared the three subgroups generated by the LPA across all six original PLQS factor-based symptom scores (figure 3). Compared with subgroup 1, 'Fatigue Cognitive' and 'Neurologic' factor-based scores were significantly higher among both subgroups 2 and 3 participants. 'Musculoskeletal Pain' was the only factor to statistically significantly differentiate all three subgroups from one another, with scores in subgroup 1 being the lowest and subgroup 2 being the highest. 'Infection-Type' and 'Ocular Disequilibrium' factor scores trended in the direction of increasing from subgroups 1 to 3. Lastly, 'Mood-Related' factor scores were significantly higher among subgroup 3 participants compared with both subgroups 1 and 2, which did not differ significantly from each other.

The results of detailed demographic, clinical, laboratory and psychosocial characteristic comparisons by subgroup are presented in table 2. Notably, neither the percentage male ( $\mathrm{q} \geq 0.887$ for all pair-wise comparisons) nor LEC total score ( $\mathrm{q} \geq 0.615$ for all pair-wise comparisons) was statistically significantly different across subgroups. Participants in subgroup 1, which generally included those with lower symptom factor-based scores, also reported lower rates of being on disability than the other two groups and had higher CDSE scores. Subgroup 2 was found to have higher blood pressure, and a higher 


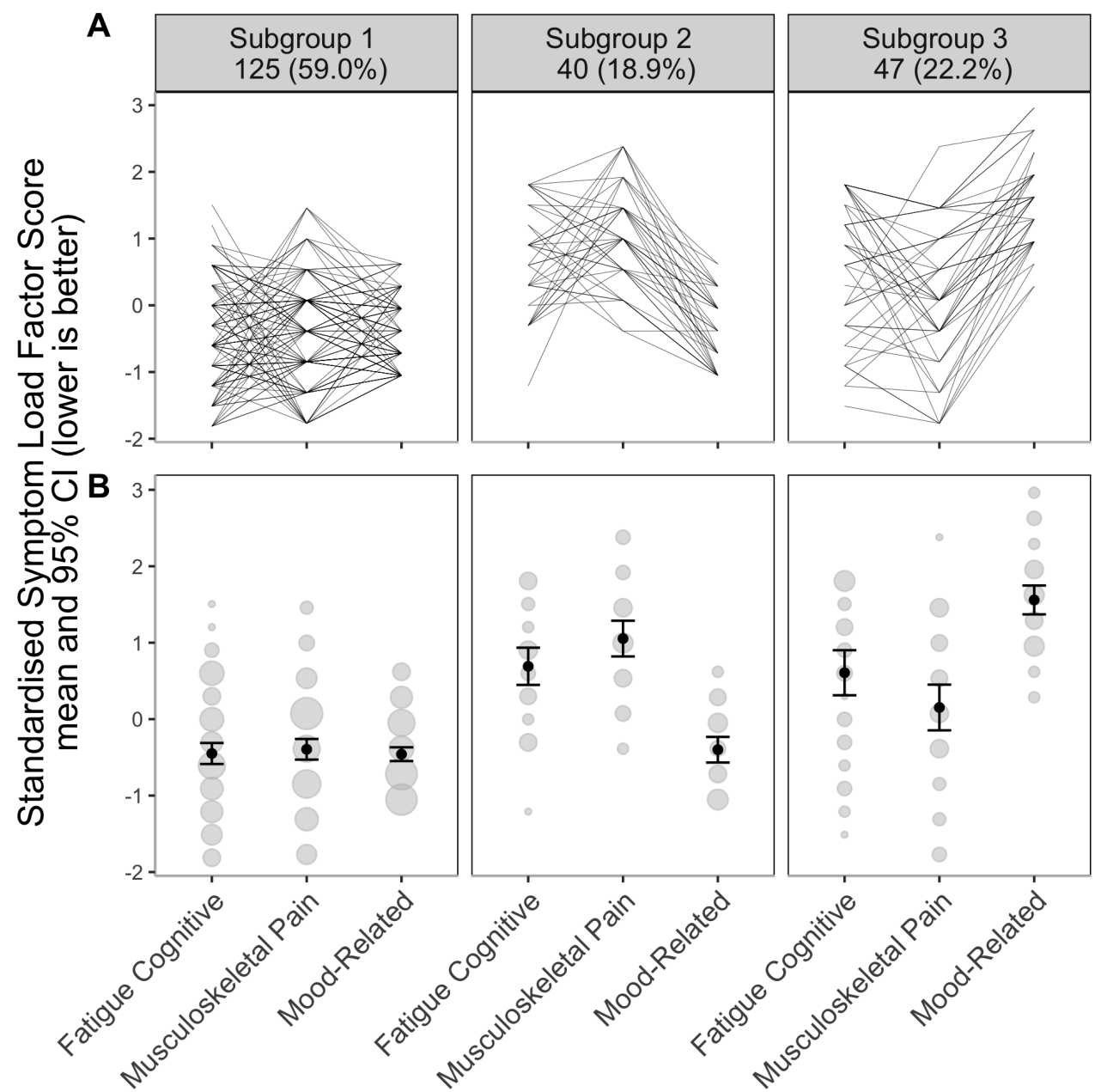

Figure 2 Three subgroups of participants identified based on latent profile analysis (A,B).

percentage of participants with an abnormal $\mathrm{C}$ reactive protein than subgroup 1.

Overall, participants in subgroup 3 were younger, with a lower percentage reporting an annual household income $>$ US $\$ 100000$. This group was also found to have a median illness duration of almost a year longer than the other two groups, and a higher percentage who reported prior intravenous antibiotic treatment. Consistent with the pattern of symptom reporting in the factor-based
PLQS scores, subgroup 3 had significantly worse BDIC/A scores than the other two subgroups. On the BFI, subgroup 3 had significantly lower scores in the conscientiousness and emotional stability domains than the other two subgroups.

Those comorbid diagnoses occurring with at least 5\% prevalence in the sample as a whole are also reported in table 2. No statistically significant differences were found for any of the conditions with the exception that

\begin{tabular}{l|c|c|c|c|c|c} 
& $\begin{array}{c}\text { Subgroup } \\
1\end{array}$ & $\begin{array}{c}\text { Subgroup } \\
2\end{array}$ & Subgroup & q-value & q-value & q-value \\
& 1 & 1 vs. 2 & 1 vs. 3 & 2 vs. 3 \\
\hline Fatigue Cognitive & & & & $<0.001$ & $<0.001$ & 1.000 \\
Musculoskeletal Pain & & & & $<0.001$ & 0.007 & 0.001 \\
Mood-Related & & & & 0.855 & $<0.001$ & $<0.001$ \\
Neurologic & & & & 0.007 & $<0.001$ & 0.738 \\
Infection-Type & & & & 0.346 & $<0.001$ & 0.220 \\
Ocular Disequilibrium & & & & 0.224 & $<0.001$ & 0.035
\end{tabular}

Figure 3 Participant subgroup differences in median standardised symptom factor-based scores, depicted as a heat map. The higher the score, the higher the severity of reported symptoms within each factor. 
Table 2 Participant subgroup comparisons across demographic, clinical laboratory and psychosocial characteristics*

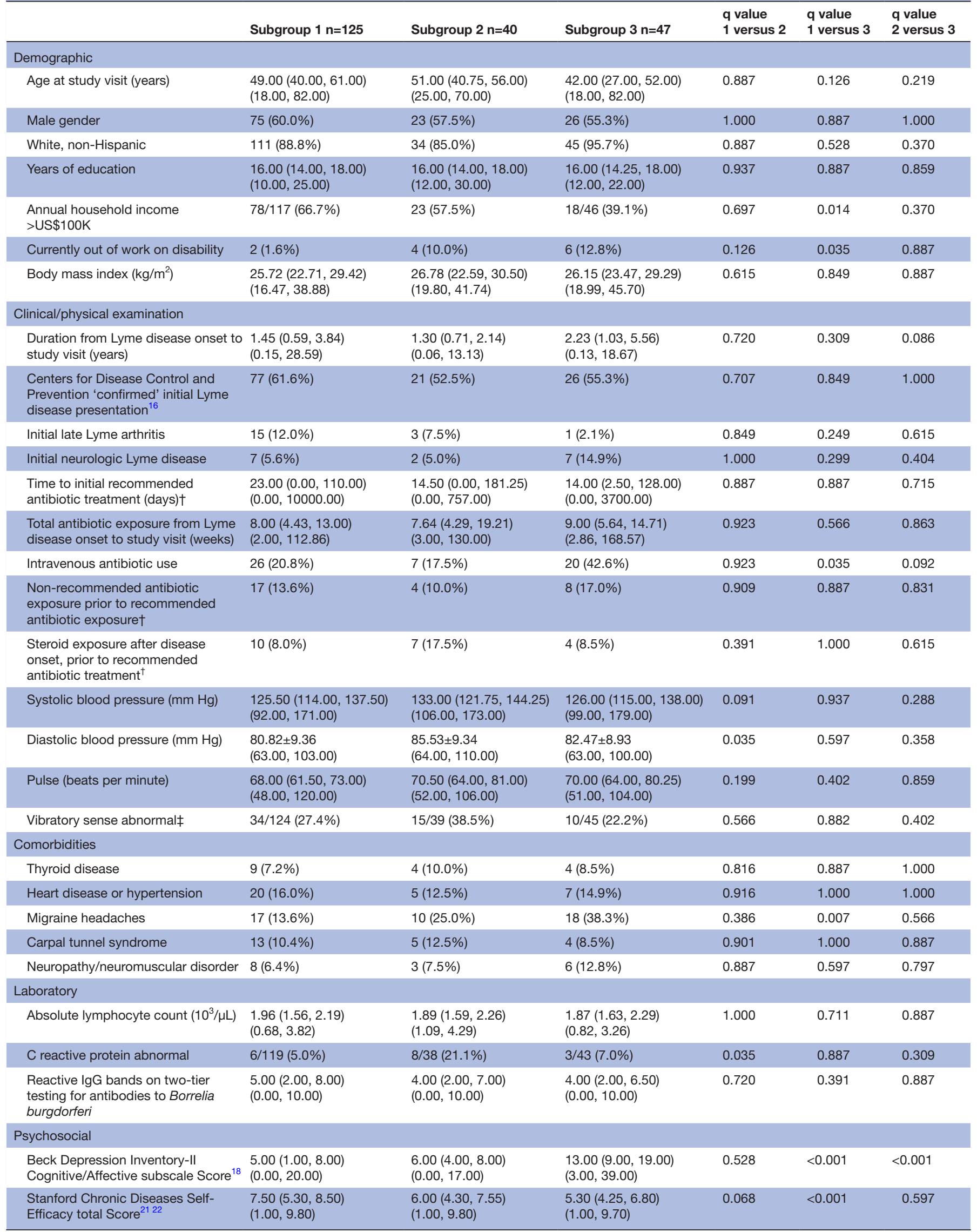




\begin{tabular}{|c|c|c|c|c|c|c|}
\hline $\begin{array}{l}\text { Big Five Inventory: Extraversion } \\
\text { Score }\end{array}$ & $\begin{array}{l}3.38(2.75,3.88) \\
(1.38,5.00)\end{array}$ & $\begin{array}{l}3.44(3.00,3.91) \\
(1.63,4.88)\end{array}$ & $\begin{array}{l}3.13(2.56,3.63) \\
(1.75,5.00)\end{array}$ & 0.797 & 0.527 & 0.355 \\
\hline $\begin{array}{l}\text { Big Five Inventory: } \\
\text { Conscientiousness Score }\end{array}$ & $\begin{array}{l}4.00(3.56,4.44) \\
(2.22,5.00)\end{array}$ & $\begin{array}{l}4.05(3.67,4.44) \\
(2.22,4.89)\end{array}$ & $\begin{array}{l}3.67(3.28,4.11) \\
(1.56,4.89)\end{array}$ & 0.887 & 0.020 & 0.035 \\
\hline $\begin{array}{l}\text { Big Five Inventory: Emotional } \\
\text { Stability Score }\end{array}$ & $\begin{array}{l}3.63(3.13,4.10) \\
(1.38,5.00)\end{array}$ & $\begin{array}{l}3.75(3.22,4.25) \\
(2.50,5.00)\end{array}$ & $\begin{array}{l}2.63(1.82,3.25) \\
(1.00,4.63)\end{array}$ & 0.668 & $<0.001$ & $<0.001$ \\
\hline Big Five Inventory: Openness Score & $\begin{array}{l}3.70(3.30,4.20) \\
(2.30,5.00)\end{array}$ & $\begin{array}{l}3.90(3.40,4.32) \\
(2.70,4.90)\end{array}$ & $\begin{array}{l}3.80(3.30,4.10) \\
(1.20,4.80)\end{array}$ & 0.495 & 1.000 & 0.615 \\
\hline
\end{tabular}

${ }^{*}$ Data from categorical variables are presented as count (\%). Data from normally distributed continuous variables are presented as mean \pm SD (range) and from continuous variables not normally distributed as median (25th percentile, 75th percentile) (range). Proportions were calculated based on non-missing data and may not add to $100 \%$ because of rounding. Missing data are as follows: years of education, 1 (0.5\%); annual household income, 9 (4.2\%); body mass index, 18 (8.5\%); systolic blood pressure, 5 (2.4\%); diastolic blood pressure, 4 (1.9\%); pulse, 3 (1.4\%); vibratory sense, 4 (1.9\%); absolute lymphocyte count, 2 (0.9\%); C reactive protein, 12 (5.7\%); IgG reactive bands, 1 (0.5\%); Beck Depression Inventory-II Cognitive/Affective Score, 1 (0.5\%); Stanford Chronic Diseases Self-Efficacy Score, 1 (0.5\%); Big Five Inventory, 3 (1.4\%).

tRecommended antibiotic regimens were considered any of the following: Doxycycline $100 \mathrm{mg}$ two times per day for $\geq 10$ days, tetracycline $500 \mathrm{mg}$ three times a day for $\geq 14$ days, ceftin $500 \mathrm{mg}$ two times per day for $\geq 14$ days, ceftriaxone $2 \mathrm{~g} /$ day for $\geq 14$ days. Other drugs, or lower doses or durations were considered non-recommended antibiotic regimes. łBelow age-adjusted normal vibration threshold values in either upper (distal interphalangeal joint of the index finger) or lower (interphalangeal joint of the hallux) extremities on either right or left side using a Rydel-Seiffer $64 \mathrm{~Hz}$ tuning fork. ${ }^{26}$

participants in subgroup 3 were almost three times as likely as those in subgroup 1 to report migraine headaches. In examining differences by subgroup in SF-36 quality of life scores, we found that subgroup 2 had significantly lower PCS scores compared with the other two groups, whereas subgroup 3 had significant lower MCS scores compared with the other two groups (figure 4). This is consistent with the pattern of symptom reporting in the factor-based scores which differentiated the three groups.

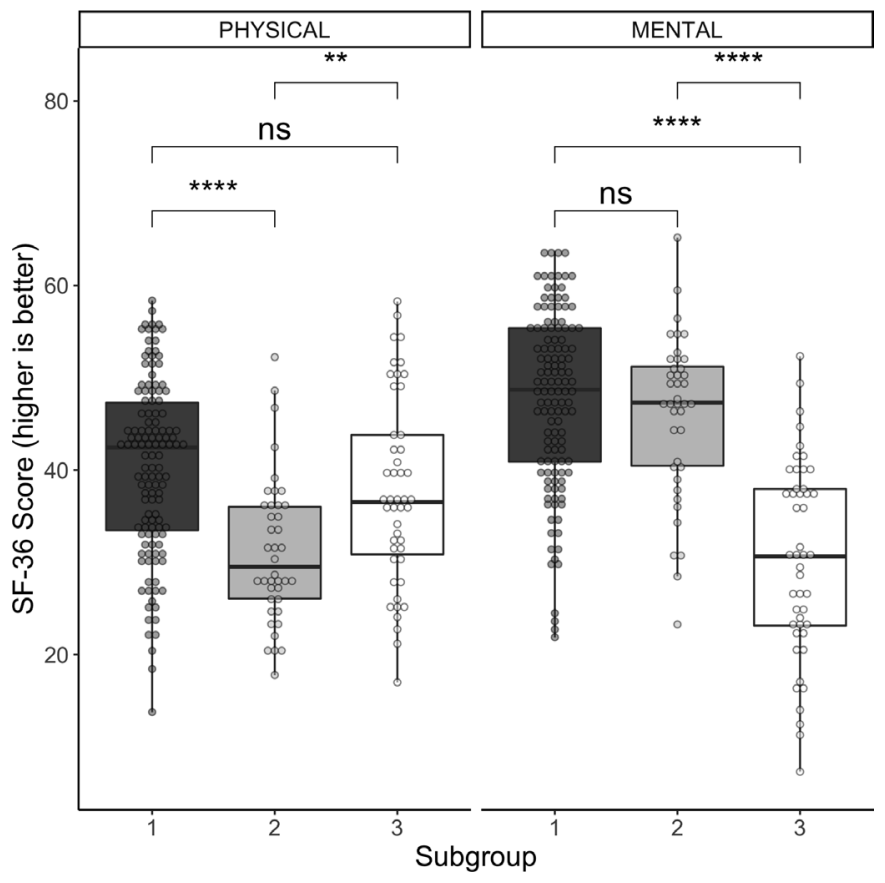

Figure 4 Short-Form Health Survey-36 (SF-36) healthrelated quality-of-life physical and mental component scores ${ }^{19}$ for the three patient subgroups. ns=not significant; ${ }^{*} \mathrm{p}<0.05 ;{ }^{\star *} \mathrm{p}<0.01 ;{ }^{\star \star *} \mathrm{p}<0.001 ;{ }^{* \star \star \star} \mathrm{p}<0.0001$.

\section{DISCUSSION}

PTLD is a complex illness which is characterised by a wide range of clinical symptoms that can significantly impact quality of life for many patients. ${ }^{810-12}$ The aim of this study was to examine heterogeneity in symptom reporting in order to ultimately identify and characterise clinically relevant patient subgroups. Using our PLQS Questionnaire, we first identified six symptom-based factors through EFA analysis. The relational structure of these results had overall clinical face validity, with symptoms clustering in seemingly physiologically relevant rather than randomly distributed ways. For example, all three cognitive symptoms loaded onto the same factor, as did joint pain, muscle pain and joint swelling. Furthermore, the six factors we identified represent commonly recognised domains in the clinical phenotype of PTLD.

Although the analyses and the measure differed, results from our EFA were generally consistent with those from a recent study with some participant sample overlap, which aimed to validate the General Symptom Questionnaire-30 (GSQ-30) in PTLD. ${ }^{30}$ One noticeable difference was that fatigue loaded with the musculoskeletal pain factor in the GSQ-30 study rather than with cognitive symptoms, as it did in the current study. This suggests that fatigue in PTLD could arise from multiple sources including pain, the central nervous system, or muscle weakness. Similarly, insomnia may also be a multifactorial symptom, as it showed low loading $(0.32)$ to the 'Infection-Type' factor in the current study, with significant cross-loading to the 'Fatigue Cognitive', 'Musculoskeletal Pain' and 'Mood-Related' factors.

Several additional symptom factor loadings were informative as well. Neck pain is relatively common in the general population, ${ }^{31}$ however it is reported with greater frequency and severity in this sample population compared with controls, ${ }^{8}$ and the cause is unknown. Given that neck pain 
loaded the strongest onto the 'Neurologic' factor, with the second strongest loading to 'Fatigue Cognitive' and not 'Musculoskeletal Pain', we hypothesise the potential for a neurologic rather than arthritic origin. We also found that difficulty breathing and heart palpitations loaded onto the 'Mood-Related' factor, implying that this constellation of symptoms may result from a common pathway such as autonomic nervous system activation or central sensitisation ${ }^{32}$ rather than specific cardiac or pulmonary pathology. Alternatively, anxiety and other mood-related symptoms could result secondary to experiencing these types of distressing physiologic symptoms. The hypothetical relational constructs we uncovered using EFA may shed light on, but not necessarily equate to, distinct biological mechanisms resulting in symptoms. Some symptoms may have a composite underlying mechanism, some may correlate with each another despite different mechanisms and some distinct factors could represent different subtypes of a shared general mechanism.

We then used a subset of the symptom-based factors in an LPA analysis to ultimately identify three patient subgroups corresponding to specific symptom profiles. This subgroup classification was prominently differentiated first by overall severity of symptom reporting, where high and low symptom reporters were identified. We plan to investigate factors associated with severity in the sample as a whole in future multivariate analyses. It is important to clarify that symptom severity in the current study is relative to this study sample of participants with PTLD and not the general population; we have previously shown a higher symptom burden in a subset of this sample of patients with PTLD compared with nonLyme infected controls. ${ }^{8}$

Similar to our previous GSQ-30 study ${ }^{30}$ we conclude that morbidity in this population can exist above and beyond the effects of mood-related symptoms. Indeed, in our EFA analysis an independent 'Mood-Related' factor was formed whose symptoms failed to load with other core symptoms of PTLD such as fatigue, pain and cognitive difficulty. This is also supported by the pattern of symptom factor-based score reporting in subgroup 2. This subgroup had the highest 'Musculoskeletal Pain' factor-based scores; however, their 'Mood-Related' factor-based scores remained relatively low, similar to those of subgroup 1 . This pattern also suggests that mood-related symptoms in PTLD may be more likely to be associated with fatigue or cognitive symptoms than with pain. Moreover, although fatigue/cognitive, mood-related and pain symptoms all formed discrete factors in our analysis, 'Mood-Related' factor scores were more strongly correlated with 'Fatigue Cognitive' than they were with 'Musculoskeletal Pain' scores ( 0.41 vs 0.21 , respectively).

We did define a subset of our sample $(22.2 \%$, subgroup 3) who overall reported significantly higher 'Mood-Related' factor-based scores relative both to the other two subgroups and to their other symptom factor-based scores. Comparing subgroups across a variety of domains suggests several possible explanations for this finding. First, despite being younger, participants in subgroup 3 had a longer illness duration, as abstracted from their medical record. We would hypothesise that the effects of a chronic, often functionally impairing illness on mood would both compound over time and be more pronounced among younger patients. Second, subgroup 3 also endorsed lower self-efficacy in managing their illness. This is unsurprising, as lower self-efficacy has been found to be associated with a higher degree of mood symptoms in a number of studies. ${ }^{33}{ }^{34}$ Furthermore, participants in subgroup 3 also scored lower on the conscientiousness and emotional stability dimensions of the BFI, although additional research is warranted to explore the complex construct of personality among patients with PTLD. In sum, our findings suggest that participants in subgroup 3 may have been more psychologically vulnerable to the effects of a significant chronic illness over time when they first encountered Lyme disease. Indeed, many of the psychosocial variables that we measured have been shown to impact illness and resilience in other similar chronic disease populations. ${ }^{35-37}$

Finally, our data also suggest that participants with prior neurologic pathology may be over-represented in subgroup 3. Although the subgroup comparisons were not statistically significant, we observed that these participants had almost three times the rate of prior neurologic Lyme disease (cranial nerve palsy, neuropathy, meningitis or encephalitis), as abstracted from their medical record, compared with the other two groups. This is consistent with the higher rate of prior intravenous antibiotic treatment in this group as well. We also found that participants in subgroup 3 were significantly more likely to report a comorbid diagnosis of migraines. In post-hoc analyses, the diagnosis of migraine predated the Lyme disease onset for $57 \%$ of those in subgroup 3 with migraine. It is possible that pre-existing neurologic vulnerabilities, such as a history of migraine and/or frank neurologic Lyme disease, are associated with a post-treatment phenotype that encompasses an increase in mood-related symptoms. ${ }^{38}$ Although, per the IDSA case definition, we excluded participants with major psychiatric illness, Lyme disease has been associated with a range of neurologic and neuropsychiatric symptoms. ${ }^{39}$ Strikingly, although female gender ${ }^{40}$ and greater exposure to prior stressful life events ${ }^{42}$ have both been associated with higher mood symptoms in a number of studies, we did not observe that these participants were any more likely to report heightened mood-related symptoms when faced with similar physical symptom levels.

Our study does have limitations. We ensured greater specificity of our findings to patients whose current illness is more evidently linked to B. burgdorferi exposure by operationalising a narrow research definition of PTLD as eligibility criteria for inclusion into our sample. However, this specificity may also limit generalisability of our findings to a larger population of patients with persistent symptoms following treatment for Lyme disease, especially atypical early presentations not meeting CDC criteria. It is possible that different eligibility criteria, or different patient samples drawn from other regions of the USA, may have different results. Given the relatively high median household income of our sample, which may have resulted from the geographic location and 
specialty referral-based nature of our clinic, it will also be important to understand if our findings are generalisable across a broader income range. Furthermore, we relied on self-report symptom data for these analyses, which are subject to response bias as well as individual variation in perception of symptom severity. ${ }^{43}$

Finally, when applying EFA, Pearson's correlation was used for data from a 4-point Likert scale, which does not satisfy the assumption of a multivariate normal distribution. A nonconvergence issue prevented us from using the more appropriate polychoric correlation. This could lead to spurious multidimensionality and biased factor loadings. ${ }^{44}$ However, EFA conceptually met the needs of our research aim, and the results based on Pearson's correlation matrix exhibited meritorious factorability and produced results with satisfactory performance measures. We also followed recommendations to improve our EFA for ordinal data, ${ }^{45}$ such as using parallel analysis-based methods for factor retention decision and oblique rotation method. In addition, the main structure of the EFA results is largely consistent with an exploratory symptom clustering analysis we conducted using Kendall's Tau-b, which is non-parametric and is appropriate for ordinal variables.

Reproducibility of the subgroup analysis may be affected by necessary methodological decisions made during the analytic process, including: the scale of the data, the inclusion of a large number of symptoms in the analysis and the statistical and clinical criteria used during the model selection process. However, the approaches we employed were chosen to achieve as high a degree of theoretical soundness and feasibility as possible. These approaches, in conjunction with the relatively large sample of participants with PTLD that we were able to draw on for this analysis, allowed for clear and concise interpretability of data.

This analysis represents one of the first to identify and characterise potentially clinically relevant patient subgroups in PTLD. This is important as it may serve as an initial step towards engaging with the heterogeneity in symptom reporting that has long been observed among patients with this condition. Furthermore, in the future it may lead to more targeted interventions or other novel treatment approaches to address the varied and/or multiple factors which contribute to illness perpetuation in PTLD.

Correction notice This article has been corrected since it first published. The provenance and peer review statement has been included.

Acknowledgements We gratefully acknowledge Dr Kristian Nitsch and Dr Pegah Touradji for their assistance reviewing the manuscript, and Cheryl Novak and Erica Mihm for their assistance conducting participant study visits.

Contributors AWR, TY and JNA all contributed to the conception and design of this study. TY and AWR conducted the data management and statistical analyses. AWR, TY and JNA drafted, revised and gave final approval of the manuscript for publication.

Funding This work was supported by a grant from the Steven and Alexandra Cohen Foundation (\#122279). The funding organisation had no role in any of the following: design and conduct of the study; data collection, analysis or interpretation; preparation, review or approval of the manuscript; decision to submit for publication.

Competing interests None declared.
Patient consent for publication Not required.

Provenance and peer review Not commissioned; externally peer reviewed.

Data availability statement Data are available upon reasonable request. De-identified participant data are available upon reasonable request to the corresponding author.

Supplemental material This content has been supplied by the author(s). It has not been vetted by BMJ Publishing Group Limited (BMJ) and may not have been peer-reviewed. Any opinions or recommendations discussed are solely those of the author(s) and are not endorsed by BMJ. BMJ disclaims all liability and responsibility arising from any reliance placed on the content. Where the content includes any translated material, BMJ does not warrant the accuracy and reliability of the translations (including but not limited to local regulations, clinical guidelines, terminology, drug names and drug dosages), and is not responsible for any error and/or omissions arising from translation and adaptation or otherwise.

Open access This is an open access article distributed in accordance with the Creative Commons Attribution Non Commercial (CC BY-NC 4.0) license, which permits others to distribute, remix, adapt, build upon this work non-commercially, and license their derivative works on different terms, provided the original work is properly cited, appropriate credit is given, any changes made indicated, and the use is non-commercial. See: http://creativecommons.org/licenses/by-nc/4.0/.

ORCID iD

Alison W Rebman http://orcid.org/0000-0002-2648-0896

\section{REFERENCES}

1 Steere AC, Strle F, Wormser GP, et al. Lyme borreliosis. Nat Rev Dis Primers 2016;2:16090.

2 Stone BL, Tourand Y, Brissette CA. Brave new worlds: the expanding universe of Lyme disease. Vector-Borne and Zoonotic Diseases 2017;17:619-29.

3 Wormser GP, Dattwyler RJ, Shapiro ED, et al. The clinical assessment, treatment, and prevention of Lyme disease, human granulocytic anaplasmosis, and babesiosis: clinical practice guidelines by the infectious diseases Society of America. Clin Infect Dis 2006;43:1089-134.

4 Wormser GP. Hematogenous dissemination in early Lyme disease. Wien Klin Wochenschr 2006;118:634-7.

5 Rebman AW, Aucott JN. Post-Treatment Lyme disease as a model for persistent symptoms in Lyme disease. Front. Med. 2020;7.

6 Hickie I, Davenport T, Wakefield D, et al. Post-infective and chronic fatigue syndromes precipitated by viral and non-viral pathogens: prospective cohort study. BMJ 2006;333:575.

7 Nath A. Long-Haul COVID. Neurology 2020;95:559-60.

8 Rebman AW, Bechtold KT, Yang T, et al. The clinical, symptom, and quality-of-life characterization of a well-defined group of patients with posttreatment Lyme disease syndrome. Front. Med. 2017;4:224.

9 Aucott JN. Posttreatment Lyme disease syndrome. Infect Dis Clin North Am 2015;29:309-23.

10 Lobraico J, Butler A, Petrini J, et al. New insights into stages of Lyme disease symptoms from a novel hospital-based registry. J Prim Care Community Health 2014;5:284-7.

11 Zomer TP, Barendregt JNM, van Kooten B, et al. Non-Specific symptoms in adult patients referred to a Lyme centre. Clin Microbiol Infect 2019;25:67-70.

12 Klempner MS, Hu LT, Evans J, et al. Two controlled trials of antibiotic treatment in patients with persistent symptoms and a history of Lyme disease. N Engl J Med 2001;345:85-92.

13 Mukherjee S, Mez J, Trittschuh EH. Genetic data and cognitively defined late-onset Alzheimer's disease subgroups. Mol Psychiatry 2018.

14 Curtis C, Shah SP, Chin S-F, et al. The genomic and transcriptomic architecture of 2,000 breast tumours reveals novel subgroups. Nature 2012;486:346-52.

15 Bellato E, Marini E, Castoldi F, et al. Fibromyalgia syndrome: etiology, pathogenesis, diagnosis, and treatment. Pain Res Treat 2012;2012:1-17.

16 Centers for Disease Control and Prevention. Lyme disease (Borrelia burgdorferi) 2011 case definition. Available: https://wwwn.cdc.gov/ nndss/conditions/lyme-disease/case-definition/2011/

17 Beck AT, Steer RA, Brown GK. Beck Depression Inventory. 2nd ed. San Antonio: The Psychological Corporation, 1996.

18 Storch EA, Roberti JW, Roth DA. Factor structure, concurrent validity, and internal consistency of the beck depression inventory?second 
edition in a sample of college students. Depress Anxiety 2004;19:187-9.

19 Ware JE, Kosinski M, Dewey JE. How to score version 2 of the SF-36 health survey. Quality Metric Inc: Lincoln, RI:, 2000.

20 Gray MJ, Litz BT, Hsu JL, et al. Psychometric properties of the life events checklist. Assessment 2004;11:330-41.

21 Lorig KR, Sobel DS, Ritter PL. Effect of a self-management program on patients with chronic disease. Eff Clin Pr 2001;4:256-62 http:// www.ncbi.nlm.nih.gov/pubmed/11769298

22 Ritter PL, Lorig K. The English and Spanish self-efficacy to manage chronic disease scale measures were validated using multiple studies. J Clin Epidemiol 2014;67:1265-73.

23 John OP, Naumann LP, Soto CJ. Paradigm shift to the integrative Big Five Trait taxonomy: History, measurement, and conceptual issues. In: John OP, Robins RW, Pervin LA, eds. Handbook of personality: theory and research. New York, NY: Guilford Press, 2008: 114-58.

24 John OP, Donahue EM, KR L. The big five Inventory-Versions $4 A$ and 54. Berkeley, CA, 1991.

25 Benet-Martínez V, John OP. Los Cinco Grandes across cultures and ethnic groups: Multitrait-multimethod analyses of the big five in Spanish and English. J Pers Soc Psychol 1998;75:729-50.

26 Martina IS, van Koningsveld R, Schmitz PI, et al. Measuring vibration threshold with a graduated tuning fork in normal aging and in patients with polyneuropathy. European inflammatory neuropathy cause and treatment (INCAT) group. J Neurol Neurosurg Psychiatry 1998;65:743-7 https://www.ncbi.nlm.nih.gov/pmc/articles/ PMC2170371/pdf/v065p00743.pdf

27 Briggs NE, MacCallum RC. Recovery of weak common factors by maximum likelihood and ordinary least squares estimation. Multivariate Behav Res 2003;38:25-56.

28 Storey J, Bass A, Dabney A. qvalue: Q-value estimation for false discovery rate control. $\mathrm{R}$ package version 2.22.0, 2020. Available: http://github.com/jdstorey/qvalue

29 Centers for Disease Control and Prevention. Lyme disease maps: most recent year. Available: https://www.cdc.gov/lyme/ datasurveillance/maps-recent.html [Accessed 28 Aug 2019].

30 Fallon BA, Zubcevik N, Bennett C, et al. The general symptom Questionnaire-30 (GSQ-30): a brief measure of multi-system symptom burden in Lyme disease. Front. Med. 2019;6:283.

31 Safiri S, Kolahi A-A, Hoy D, et al. Global, regional, and national burden of neck pain in the general population, 1990-2017: systematic analysis of the global burden of disease study 2017 . BMJ 2020;12:m791.
32 Batheja S, Nields JA, Landa A, et al. Post-Treatment Lyme syndrome and central sensitization. J Neuropsychiatry Clin Neurosci 2013;25:176-86.

33 Muris P. Relationships between self-efficacy and symptoms of anxiety disorders and depression in a normal adolescent sample. Pers Individ Dif 2002;32:337-48.

34 Shnek ZM, Foley FW, LaRocca NG, et al. Helplessness, self-efficacy, cognitive distortions, and depression in multiple sclerosis and spinal cord injury. ann. behav. med. 1997;19:287-94.

35 Galvez-Sánchez CM, Montoro CI, Duschek S, et al. Depression and trait-anxiety mediate the influence of clinical pain on health-related quality of life in fibromyalgia. J Affect Disord 2020;265:486-95.

36 Torres X, Bailles E, Valdes M, et al. Personality does not distinguish people with fibromyalgia but identifies subgroups of patients. Gen Hosp Psychiatry 2013;35:640-8.

37 Van Liew C, Leon G, Neese M, et al. You get used to it, or do you: symptom length predicts less fibromyalgia physical impairment, but only for those with above-average self-efficacy. Psychol Health Med 2019;24:207-20.

38 Affaitati G, Costantini R, Tana C, et al. Co-Occurrence of pain syndromes. J Neural Transm 2020;127:625-46.

39 Fallon BA, Nields JA. Lyme disease: a neuropsychiatric illness. Am J Psychiatry 1994;151:1571-83 http://www.ncbi.nlm.nih.gov/pubmed/ 7943444

40 McLean CP, Asnaani A, Litz BT, et al. Gender differences in anxiety disorders: prevalence, course of illness, comorbidity and burden of illness. J Psychiatr Res 2011;45:1027-35.

41 Albert P. Why is depression more prevalent in women? J Psychiatry Neurosci 2015;40:219-21.

42 Tennant C, events L. Life events, stress and depression: a review of recent findings. Aust N Z J Psychiatry 2002;36:173-82.

43 Broadbent E, Petrie KJ, et al. Symptom perception. In: Ayers S, Baum A, McManus C, eds. Cambridge Handbook of psychology, health and medicine. 2nd ed. Cambridge University Press, 2007: 219-23.

44 Bernstein IH, Teng G. Factoring items and factoring scales are different: spurious evidence for multidimensionality due to item categorization. Psychol Bull 1989;105:467-77.

45 Baglin J. Improving Your Exploratory Factor Analysis for Ordinal Data: A Demonstration Using FACTOR. Pract Assessment. Res Eval $2019 ; 19$. 